\title{
ACC Subduction by Mesoscales
}

\author{
V. M. CANUTO \\ NASA Goddard Institute for Space Studies, and Department of Applied Physics and Mathematics, \\ Columbia University, New York, New York \\ Y. CHENG \\ NASA Goddard Institute for Space Studies, and Center for Climate Systems Research, \\ Columbia University, New York, New York
}

(Manuscript received 18 February 2019, in final form 1 October 2019)

\begin{abstract}
The mesoscale contribution to subduction in the Southern Ocean was studied by Sallée and Rintoul in 2011 (SR11) using the following mesoscale model. The adiabatic (A) regime was modeled with the Gent-McWilliams streamfunction, the diabatic (D) regime was modeled with tapering functions, the D-A interface was taken to be at the mixed layer depth, and the mesoscale diffusivity either was a constant or was given by a $2 \mathrm{D}$ model. Since the resulting subductions were an order of magnitude smaller than the data of $\pm 200 \mathrm{~m} \mathrm{yr}^{-1}$ as reported by Mazloff et al. in 2010, SR11 showed that if, instead of the above model-dependent mesoscale diffusivities, they employed the ones reported in 2008 by Sallée et al. from surface drifter observations, the subductions compared significantly better to the data. On those grounds, SR11 suggested a 10-fold increase of the diffusivity. In this work, we suggest that, since the mesoscale diffusivity is but one component of a much large mesoscale parameterization, one should first assess the latter's overall performance followed by an assessment of the predicted Antarctic Circumpolar Current (ACC) subduction. We employ the mesoscale model formulated by Canuto et al. in 2018 and 2019 that includes recent theoretical and observational advances and that was assessed against a variety of data, including the output of 17 other OGCMs. The ACC diffusivities compare well to drifter data from Sallée et al., and the ACC subduction rates are in agreement with the data.
\end{abstract}

\section{Sallée-Rintoul model}

Subduction irreversibly transfers water masses from the mixed layer depth $H$ to the interior thermocline. The form of the subduction rate $S_{b}$ reads as follows (Cushman-Roisin 1987; Marshall 1997):

$$
\begin{aligned}
S_{b} & =\frac{\partial H}{\partial t}+\underbrace{\overline{\mathbf{u}} \cdot \nabla H+\bar{w}}_{\text {mean }}+\underbrace{\mathbf{u}^{+} \cdot \nabla H+w^{+}}_{\text {eddy }} \\
& =\frac{\partial H}{\partial t}+S_{b}(\text { mean })+S_{b} \text { (eddy) } .
\end{aligned}
$$

Sloyan and Rintoul (2001), Sallée et al. (2010), and Sallée and Rintoul (2011, hereinafter SR11) computed $S_{b}$ (eddy), and Sallée et al. (2010, their section 4b) concluded that the eddy component "plays an order one role in the overall subduction in the Southern Ocean." SR11 employed the following four-part model: 1) The adiabatic (A) regime

\footnotetext{
Corresponding author: V. M. Canuto, vmcanuto@gmail.com
}

was treated using the Gent-McWilliams (GM) streamfunction $\boldsymbol{\Psi}_{A}$ (Gent and McWilliams 1990):

$$
\Psi_{A}=-\kappa_{M} \mathbf{s} \times \mathbf{e}_{z},
$$

where $\kappa_{M}$ is the mesoscale diffusivity, $\mathbf{s}\left(=-N^{-2} \nabla_{H} \bar{b}\right)$ is the slope of the isopycnals, and $\mathbf{e}_{z}=(0,0,1)$. 2) The diabatic (D) regime was parameterized as an extension of the A regime using

$$
\boldsymbol{\Psi}_{D}=-\kappa_{M} T(x, y, z) \mathbf{s} \times \mathbf{e}_{z},
$$

where the tapering function $T(x, y, z)$ was assumed to depend only on $z$ with the boundary conditions $T(0)=0$ and $T(\mathrm{~A}-\mathrm{D}$ interface $)=1.3$ ) The A-D interface was taken at $H$. 4) The mesoscale diffusivity was taken to be

$$
\kappa_{M}=10^{3} \mathrm{~m}^{2} \mathrm{~s}^{-1}
$$

and 2D (Visbeck et al. 1997). Figure 4 of SR11 shows that the predicted subduction rates are an order of 
magnitude smaller than the observed value of $\pm 200 \mathrm{~m} \mathrm{yr}^{-1}$ (Mazloff et al. 2010), which is hereinafter referred to as the Southern Ocean state estimate (SOSE). On the other hand, use of the diffusivities derived by Sallée et al. (2008) from surface drifter observations, yielded subductions that compared significantly better to the data. On those grounds, SR11 suggested a 10-fold increase of the mesoscale diffusivity in Eqs. (1.2)-(1.4).

Since the mesoscale diffusivity is but one component of a complete mesoscale parameterization, we suggest that the latter should first be assessed on its overall performance, the Antarctic Circumpolar Current (ACC) subduction being one of the tests. We employ the mesoscale models presented in Canuto et al. (2018a, hereinafter C18) and Canuto et al. (2019, hereinafter C19) that include recent theoretical and observational advances and that were assessed against a variety of data and the outputs of 17 other ocean general circulation models (OGCMs) (Griffies et al. 2009). The present model yields two main results: the ACC diffusivities compare well to those from drifter data (Sallée et al. 2008) and the ACC subduction rates are of the same magnitude as the SOSE data.

\section{New mesoscale model}

For the reader's convenience, we have added appendix A with the relevant equations of $\mathrm{C} 18$ and $\mathrm{C} 19$.

\section{a. A regime}

A census of TOPEX-Poseidon (T/P) altimetry data led Chelton et al. (2011, hereinafter C11) to the conclusion that "essentially all of the observed mesoscale features are highly non-linear" which calls for a nonlinear treatment of mesoscales. Six years before C11, a nonlinear mesoscale model was proposed (Canuto and Dubovikov 2005, hereinafter CD5), but lack of data did not allow the assessment of the model's two key predictions: first, mesoscales do not travel with the mean velocity but with their own drift velocity $\mathbf{u}_{d}$, which is a barotropic variable since it is the solution of an eigenvalue problem. The $\mathrm{T} / \mathrm{P}$-based conclusions by $\mathrm{C} 11$ confirmed the prediction and defined $\mathbf{u}_{d}$ as the most germane of all the nonlinear metrics. It must be noted that $\mathbf{u}_{d}$ cannot be identified with the Rossby phase velocity resulting from linear analysis and that does not reproduce altimetry data (Klocker and Marshall 2014). Figure 1 of $\mathrm{C} 18$ shows that the form of $\mathbf{u}_{d}$ given by Eq. (2.5) of $\mathrm{C} 18$ compares well to altimetry data. Second, the eddy-induced velocity is no longer given by the GM form alone since $\mathbf{u}_{d}$ introduces a second term:

$$
\mathbf{u}^{+}=-\frac{\partial}{\partial z} \kappa_{M} \mathbf{s}-\frac{\kappa_{M}}{\sigma f r_{d}^{2}} \mathbf{e}_{z} \times\left(\overline{\mathbf{u}}-\mathbf{u}_{d}\right),
$$

where $\sigma_{t} \equiv \sigma_{t}\left(1+\sigma_{t}\right)^{-1}$ and $\sigma_{t}=O(1)$ is the turbulent Prandtl number. The implication of the new term in Eq. (2.1) was first studied in CD5 and more quantitatively in section $2 \mathrm{f}$ of $\mathrm{C} 18$, where it was shown that it lowers the amount of energy that mesoscales draw from the mean potential energy, which in turn implies that the isopycnal slopes are steeper than in the GM model (see Fig. 4 of C18). This feature becomes relevant when studying, for example, the implications of the predicted increase of the wind stress that tends to steepen the isopycnal slopes (Gent 2016). The first GM term in Eq. (2.1) becomes the full eddy-induced velocity only at the steering level where $\overline{\mathbf{u}}=\mathbf{u}_{d}$ at about 2-km depth when mesoscales comove with the mean velocity. Since Fig. 3 of $\mathrm{C} 18$ shows that above $2 \mathrm{~km} \bar{u}-u_{d}>0$ while $\bar{v}-v_{d}<0$, in the ACC where $f<0$, one has $\left[A=\kappa_{M} /\left(\sigma|f| r_{d}^{2}\right)\right]$ :

$$
u^{+}=u_{\mathrm{GM}}+A\left|\bar{v}-v_{d}\right|, \quad v^{+}=v_{\mathrm{GM}}+A\left|\bar{u}-u_{d}\right|,
$$

and thus, the new term in Eq. (2.1) enhances the eddy term $\mathbf{u}^{+} \cdot \nabla H$ and thus subduction.

\section{b. D regime}

To parameterize the $\mathrm{D}$ regime, SR11 employed a heuristic tapering function $T(x, y, z)$ whereby the streamfunction is considered an extension of the first of Eq. (1.2) in the form Eq. (1.3):

$$
\boldsymbol{\Psi}_{D}=-\kappa_{M} T(x, y, z) \mathbf{s} \times \mathbf{e}_{z},
$$

and the eddy-induced velocities read as follows:

$$
\mathbf{u}^{+}=-\frac{\partial}{\partial z}\left[\kappa_{M} T(x, y, z) \mathbf{s}\right], \quad w^{+}=\nabla_{H} \cdot\left[\kappa_{M} T(x, y, z) \mathbf{s}\right] .
$$

Although in principle $T(x, y, z)$ depends on $x, y, z$, thus far it has always been taken to be a function of $z$ only, an assumption that has the following implication. Consider the second relation in Eq. (2.4):

$$
w^{+}=\kappa_{M} \mathbf{s} \cdot \nabla_{H} T(x, y, z)+T(x, y, z) \nabla_{H} \cdot \mathbf{s} \kappa_{M} .
$$

The assumption that $T(x, y, z)$ depends only on $z$ makes the first term on the right-hand side of Eq. (2.5) vanish which affects the subduction rates. As for $T(z)$, SR11 adopted a straight line with the conditions $T(0)=0$ to ensure that $w^{+}(0)=0$ [see Eq. (1.4) of C18]. If the A-D interface is denoted by $h$, matching Eq. (2.3) with Eq. (1.2) requires that $T(h)=1$, but the choice of $h$ is not trivial. For example, Gnanadesikan et al. (2007) concluded that a tapering approach yielded OGCMs results that were "disconcerting" because of the strong 
dependence of $h$ on the isopycnal slope at that depth. Last, while tapering functions may work as a numerical device, the physical content of the $\mathrm{D}$ regime can hardly be represented that way since, as discussed in section $1 \mathrm{~b}$ of $\mathrm{C} 18$, the $\mathrm{A}$ and $\mathrm{D}$ regimes satisfy very different conservation laws, that is, potential vorticity in the A regime with an inverse energy cascade and relative vorticity in the $\mathrm{D}$ regime with enstrophy cascade.

To avoid tapering functions, one needs a parameterization of the $\mathrm{D}$ regime, which turned out to be a difficult task, as shown by the seven different heuristic parameterizations that were proposed (cited in section $1 \mathrm{~b}$ of C18). Since no unique formulation emerged, Canuto and Dubovikov (2011, hereinafter CD11) employed invariance properties and physical arguments and derived the following eddy streamfunction:

$$
\boldsymbol{\Psi}^{+}=-\frac{F_{v}(\bar{b})}{N^{2}\left|\mathbf{s}^{2}\right|} \mathbf{s} \times \mathbf{e}_{z},
$$

which is valid in both the $\mathrm{A}$ and $\mathrm{D}$ regimes; for example, in the A regime $F_{v}(\bar{b})=\kappa_{M} N^{2}\left|\mathbf{s}^{2}\right|$ yields Eq. (1.2). The vertical buoyancy flux $F_{V}(\bar{b})$ was given in Eqs. (3.1) and (3.2) of $\mathrm{C} 18$, and we rewrite it in a form that is as close as possible to the one in the A regime:

$$
\begin{gathered}
F_{V}(\bar{b})=\kappa_{M} N^{2} \mathbf{s} \cdot \mathbf{\Sigma}, \quad \mathbf{\Sigma}=\boldsymbol{\omega} \times \mathbf{e}_{z} \\
f r_{d}^{2} \boldsymbol{\omega} \equiv z \mathbf{u}_{D}+\int_{0}^{z} \mathbf{u}_{D}\left(z^{\prime}\right) d z^{\prime}
\end{gathered}
$$

where $\mathbf{u}_{D}=\overline{\mathbf{u}}-\mathbf{u}_{d}$. It must be pointed out that, using a mesoscale resolving numerical simulation, Luneva et al. (2015) presented a detailed assessment of the flux Eq. (2.7) under a variety of external forcing. Using Eq. (2.7) into Eq. (2.6), the latter acquires the form of Eq. (2.3) with the tapering function now given by the following relation:

$$
T(x, y, z)=\left|\mathbf{s}^{2}\right|^{-1} \mathbf{s} \cdot \mathbf{\Sigma},
$$

which shows that $T(x, y, z)$ is a function of $x, y, z$ that is no longer arbitrary but is given by the mesoscale model itself. Figure 7 of $\mathrm{C} 18$ shows that Eq. (2.8) yields results lower than the commonly used straight line.

\section{c. Extent of the $D$ regime}

Since the D-regime extent $h$ is not determined by a mesoscale model, SR11 [their Eq. (4)] and Sallée et al. [2010, their Eq. (11)] assumed

$$
h=H,
$$

which is not in accordance with results of numerical simulations showing that below the mixed layer the flow is still diabatic (Mensa et al. 2013; Veneziani et al. 2014; Ramachandran et al. 2014). The inadequacy of Eq. (2.9) was also discussed by Gregory (2000, their section 2). The $\mathrm{D}$ regime is characterized by strong vertical mixing due to wind stress that destabilizes the stable stratification represented by the positive square of the BruntVäisälä frequency. In the KPP vertical mixing schemes (Large et al. 1994), the strong mixing ceases at a depth where the bulk Richardson number becomes $O(1)$. Such a depth is called the boundary layer depth (HBL) and is location dependent. While the choice of HBL as the lower limit of $h$ is well motivated, it is still not sufficient since one also needs to know how deep h can be. In that respect, we suggest that $h$ should be less than the depth of the thermocline since at that depth the stratification would be too strong for the D regime to exist. We thus suggest the following heuristic expression:

$$
h=0.5\left[\mathrm{HBL}+\operatorname{depth} \text { of } \max N^{2}(z)\right] .
$$

Buckingham et al. (2017, their section 4.3.2) also suggested the existence of lower and upper bounds for $h$ that they called $H_{1,2}$; the upper bound was the depth of peak stratification as in Eq. (2.10) but the lower bound was still taken to be the mixed layer depth rather than the HBL. In Fig. 1 we plot the ratio $h / H$ in the ACC, where $h$ is computed using Eq. (2.10) and the mixed layer depth $H$ is computed from the potential density criterion $\Delta \sigma=0.03 \mathrm{~kg} \mathrm{~m}^{-3}$. The results in Fig. 1 show that in the majority of locations $h>H$ or $h \approx H$, in accordance with previous authors (e.g., Mensa et al. 2013; Veneziani et al. 2014) who found $h>H$. At the same time, the results also show that it is possible that $h<$ $H$ in some locations, as suggested by an anonymous referee.

\section{d. Mesoscale diffusivity}

The mesoscale diffusivity $\kappa_{M}$ is a key ingredient in any mesoscale parameterization, and the difficulties in determining it are demonstrated by the variety of suggestions that were made, for example, in section $3 \mathrm{c}$ of Sallée et al. (2010). Thus far, all of the suggested expressions were heuristic, and one can surmise the following time sequence of models of increasing physical content:

$$
\kappa_{M}(\text { constant }) \rightarrow \kappa_{M}(2 \mathrm{D}) \rightarrow \kappa_{M} \propto N^{2} \rightarrow \kappa_{M}(3 \mathrm{D}) .
$$

The first entry is no longer viable since it leads to noeddy saturation (Gent 2016); the 2D model is an improvement but fails to reproduce WOCE (2002) data showing the vertical structure of the eddy kinetic energy with enhanced surface values [see Fig. 1 of C19]. To account for this feature, Sallée et al. (2010) adopted the third relation in Eq. (2.11) in which $N^{2}$ was considered 

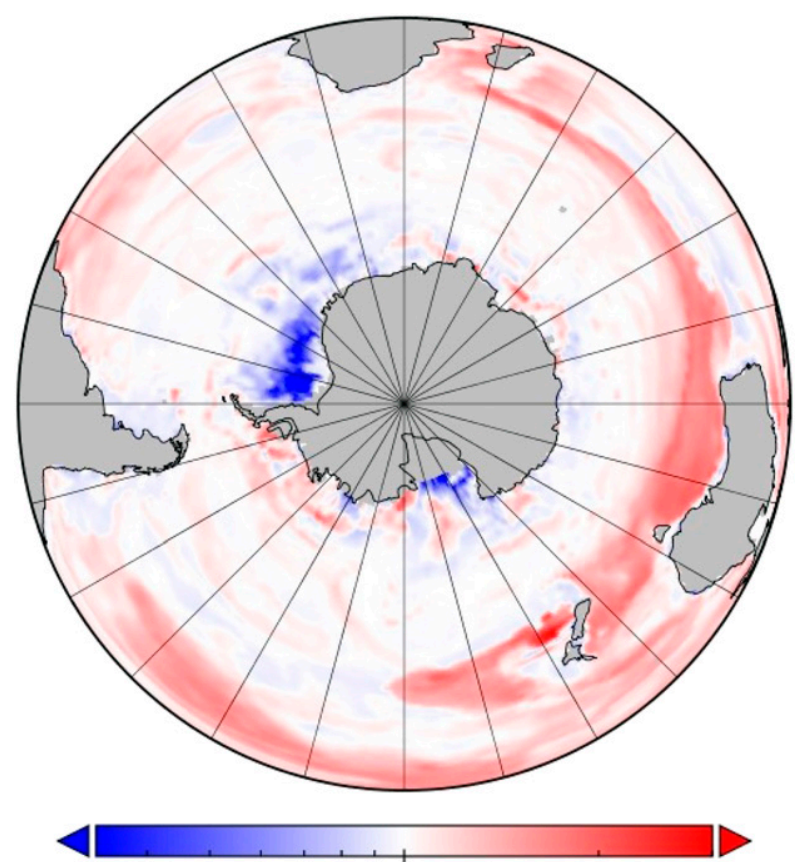

$1 / 3$ 1

\section{$h / H$}

FIG. 1. ACC map of $h$ [Eq. (2.10)] in units of the mixed layer depth $H$ computed using the potential density criterion $\Delta \sigma=$ $0.03 \mathrm{~kg} \mathrm{~m}^{-3}$. The results correspond to an average of the last 20 winters (July-September) of a 500-yr OGCM run.

to be a proxy for the eddy kinetic energy. While an improvement, it does not provide the full $x, y$ dependence shown by the T/P data (Scharffenberg and Stammer 2010), which can only be obtained by constructing the last entry in Eq. (2.11), a model of the 3D $\kappa_{M}(x, y, z)$.

The strength and reliability of any $\kappa_{M}(x, y, z)$ model depend on how accurately the key ingredient, the eddy kinetic energy $K(x, y, z)$, reproduces the WOCE (2002) data for the vertical profile and the T/P data (Scharffenberg and Stammer 2010) for the $x, y$ surface values. Canuto and Dubovikov [1996, their Eq. (24)] derived the expression for the turbulent viscosity felt by an eddy of size $\ell$ caused by all the eddies smaller than $\ell$. Section 2 of $\mathrm{C} 19$ discusses how that expression is applied to the present oceanic context and further shows how it contains the well-known mixing length theory as a particular case. The structure of the mesoscale diffusivity given by Eq. (2.5) of $\mathrm{C} 19$ is

$$
\kappa_{M}=\alpha r_{d} K^{1 / 2} \varpi\left(\mathbf{u}_{D}, K\right),
$$

where $\alpha \cong 1 / 2$ represents the departure from the mixing length theory, as explained in Eqs. (2.3) and (2.4) of C19; $r_{d}$ is the Rossby deformation radius, $K(x, y, z)$ is the $3 \mathrm{D}$ eddy kinetic energy, and $\varpi\left(\mathbf{u}_{d}, K\right)$ represents the interaction of mesoscales with the mean velocity $\overline{\mathbf{u}}$. To use Eq. (2.12), one must parameterize the eddy kinetic energy $K(x, y, z)$ and the barotropic mesoscale drift velocity:

$$
K(x, y, z)=\Gamma(z) K_{s}(x, y), \mathbf{u}_{d}(x, y) .
$$

Bates et al. (2014) determined the variables in Eq. (2.13) using data from the present climate with the resulting diffusivity shown in their Fig. 10a. Since this procedure lacks predictive power, it may not be appropriate for climate studies when future increase in wind strength may significantly change the eddy kinetic energy from today's value. To parameterize the functions in Eq. (2.13), we employ the eddy drift velocity given by Eq. (2.5) of C18 and its assessment versus T/P data shown in Fig. 1 of that paper. In $\mathrm{CD} 5$, the vertical structure of $K$ was derived to be

$$
\Gamma(z)=\left|1+a_{0}\right|^{-2}\left|a_{0}+B_{1}(z)\right|^{2},
$$

where $B_{1}(z)$ is the first baroclinic mode (Wunsch 1997), $a_{0}^{2}=\left|B_{1}\left(-H_{b}\right)\right|$ represents the barotropic contribution, and Fig. 1 of $\mathrm{C} 19$ shows the comparison of Eq. (2.14) with WOCE (2002) data in different regions. The more difficult determination of $K_{s}(x, y)$ was discussed in detail in section 4 of C19, with the result given by their Eq. (4.10) and the assessment against T/P data (Scharffenberg and Stammer 2010) shown in Figs. 9 and 10 of C19. It is relevant to point out that both vertical and horizontal components of $K(x, y, z)$ were expressed analytically. Last, the function $\varpi\left(\mathbf{u}_{d}, K\right)$ was derived to be

$$
\varpi\left(\mathbf{u}_{D}, K\right)=\left(1+\frac{\left|\mathbf{u}_{D}\right|^{2}}{K}\right)^{-1 / 2} .
$$

For small values of $\left|\mathbf{u}_{D}\right|^{2} \mathrm{~K}^{-1}$, (2.15) recovers the heuristic expression used by Bates et al. (2014). Figure 5 of C19 shows the comparison of (2.12) with North Atlantic Tracer Release Experiment (NATRE) data (Ferrari and Polzin 2005).

\section{OGCM results from the $\mathrm{C} 18$ and C19 parameterizations}

In addition to the tests discussed above, we used the new mesoscale parameterization in the GISS-ER stand-alone OGCM (see appendix B) under Core Ocean-Reference Experiment phase 1 (CORE-I) forcing (Griffies et al. 2009). The 500-yr run yielded the results in Figs. $12-17$ of $\mathrm{C} 19$ showing the global ocean temperature, the Atlantic overturning circulation, the meridional heat transport, and the Drake Passage 


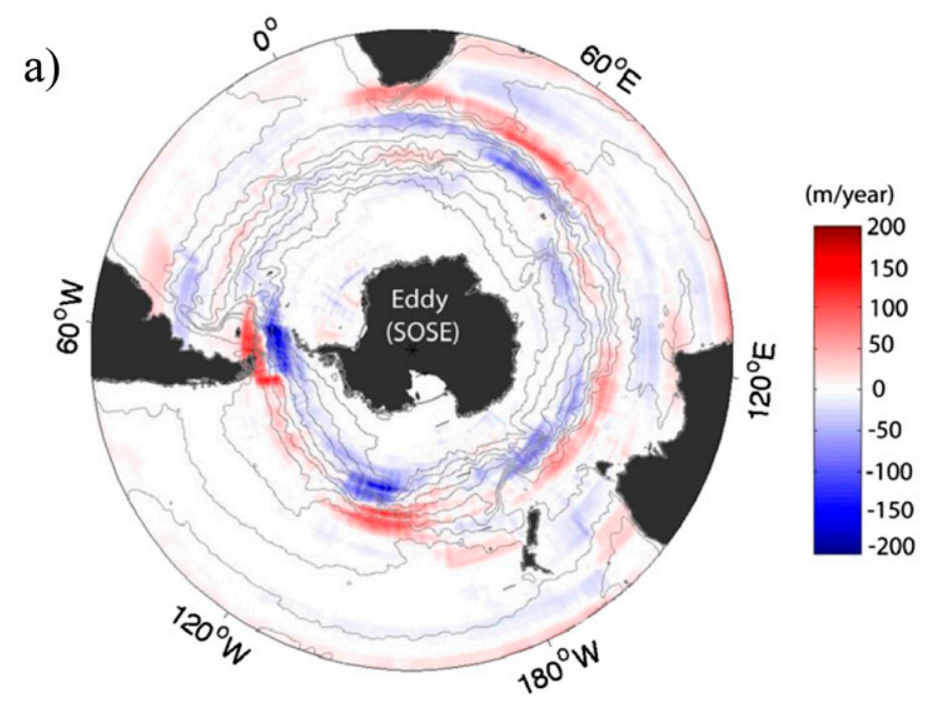

b)

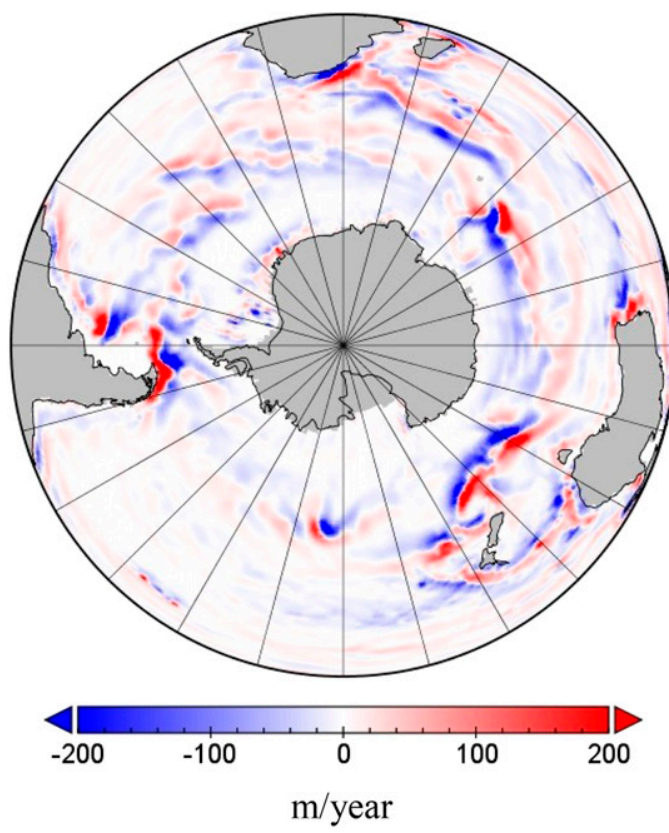

c)

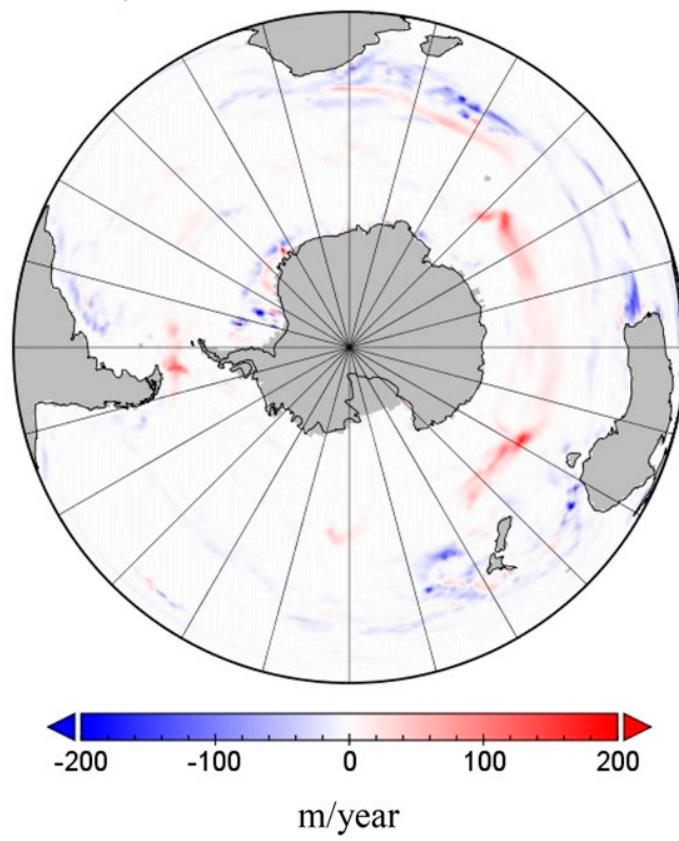

FIG. 2. (a) Subduction rates from SOSE (reproduced from SR11), (b) subduction rates from the present mesoscales model, and (c) subduction rates from the present model with $w^{+}=0$. The results correspond to an average of the last 20 winters (July-September) of a 500-yr OGCM run.

transport, all of which were compared with the results of 17 previous OGCMs. Figure 18 of $\mathrm{C} 19$ shows how the model reproduces the winter ACC mixed layer depths.

\section{Mesoscale diffusivity and subduction rates}

The mesoscale diffusivities derived by Sallée et al. (2008) using surface drifter data were larger than those used in the SR11 model and reproduced more closely the SOSE data. This motivated SR11 to suggest to boost the diffusivity in
Eqs. (1.2)-(1.4) 10-fold. Since the subduction rates we obtain shown in Fig. 2 reproduce satisfactorily the SOSE data, it remains to be shown that the mesoscale diffusivities predicted by the present model reproduce the surface drifter data. Before we do so, we need to remark that the reason to study the case in Fig. $2 \mathrm{c}$ with $w^{+}=0$ was to highlight the contribution of $w^{+}$since Hiraike et al. (2016), using an eddy-resolving simulation, reported that the $w^{+}$contribution is large; indeed, Fig. 2c shows that with $w^{+}=0$, the resulting subduction rates do not reproduce 

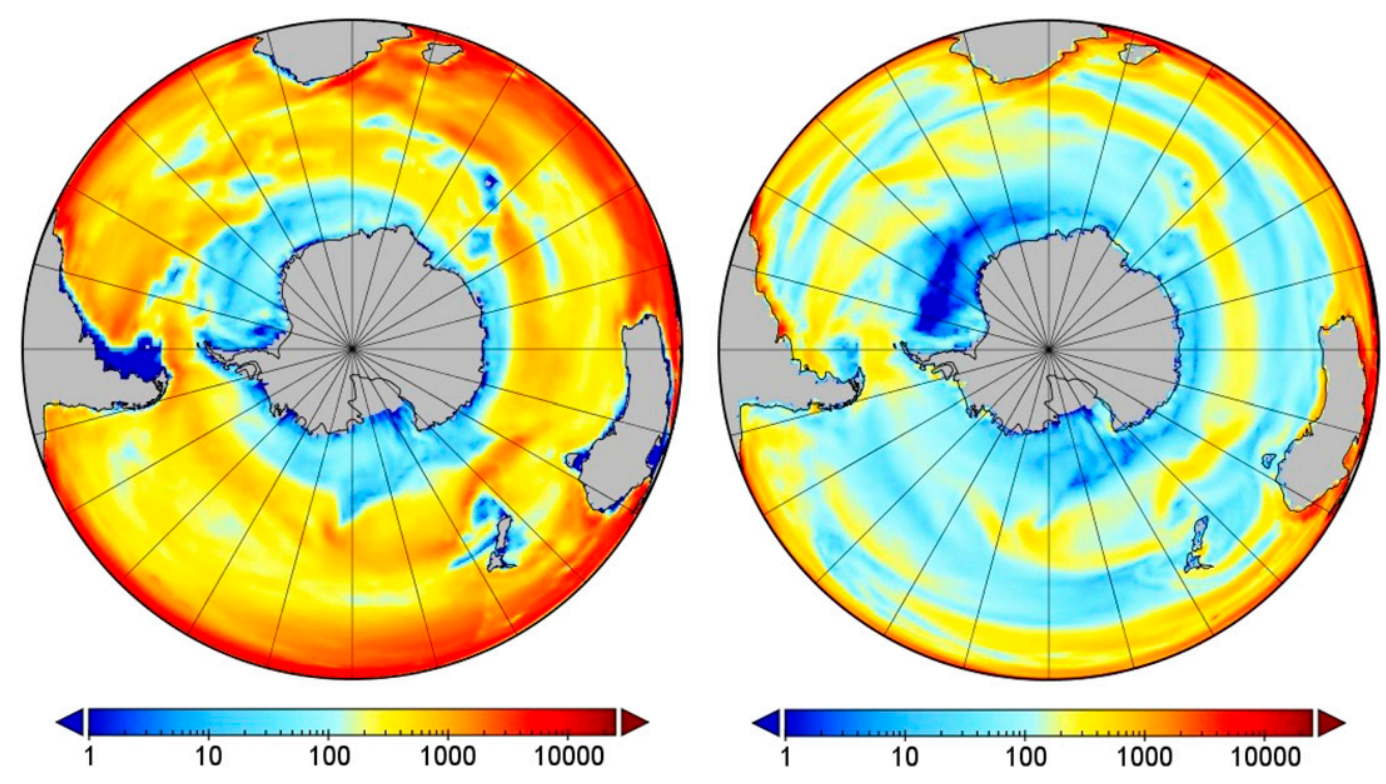

FIG. 3. Surface $\kappa_{M}\left(\mathrm{~m}^{2} \mathrm{~s}^{-1}\right)$ from the (left) 3D and (right) 2D model. The results correspond to an average of the last 20 winters (July-September) of a 500-yr OGCM run.

the SOSE data. Next, consider Fig. 3. The 3D diffusivities of this model shown in the left panel compare well to the results in Fig. 3 of Sallée et al. (2008); for completeness, the right panel shows the 2D diffusivities used by SR11.

\section{Conclusions}

The two models for the mesoscale diffusivity Eq. (1.4) employed by SR11 yielded subduction rates smaller than SOSE data by an order of magnitude. On the other hand, the mesoscale diffusivities derived by Sallée et al. (2008) from surface drifter data were larger than those in Eq. (1.4) and reproduced more closely the data. Thus, SR11 suggested boosting the diffusivity in Eq. (1.4) 10-fold. In this work, we used the mesoscale parameterizations presented in $\mathrm{C} 18$ and $\mathrm{C} 19$, whose implications were assessed against a variety of data before being used in the subduction problem that represents an additional test of the $\mathrm{C} 18$ and $\mathrm{C} 19$ parameterizations. Use of the latter reproduced satisfactorily topology (subduction equatorward and obduction poleward) and magnitudes of the SOSE data. Last, because it was previously shown (Canuto et al. 2018b) that submesoscales also produce sizeable subduction but with a topology different than that of mesoscales, a complete picture will require that mesoscales and submesoscales be considered together.

Acknowledgments. Author Canuto thanks Profs. Y. Tanaka and D. Marshall for informative correspondence. The authors thank two referees for questions that helped to sharpen our presentation. Resources supporting this work were provided by the NASA High-End Computing (HEC) Program through the NASA Center for Climate Simulation (NCCS) at the Goddard Space Flight Center.

\section{APPENDIX A}

\section{Mesoscale Parameterization for Coarse-Resolution OGCMs}

The mesoscale parameterizations in $\mathrm{C} 18$ and $\mathrm{C} 19$ is summarized as follows. The OGCMs solve the equations for the mean momentum and mean arbitrary tracers, the latter being both active tracers (such as $T, S$ ) and passive tracers (such as $\mathrm{CO}_{2}$ ) that have different parameterizations. In $\mathrm{C} 18$ and $\mathrm{C} 19$ we treated the effect of mesoscales on an arbitrary tracer since the parameterization of the mesoscale-induced momentum fluxes (Reynolds stresses) are not yet available (work is in progress). Diabatic (D) and adiabatic (A) regimes are governed by different conservation laws and have different parameterizations.

\section{a. General relations}

\section{1) D REGIME}

The equation governing a mean tracer $\bar{\tau}$ reads as follows:

$$
\partial_{t} \bar{\tau}+\overline{\mathbf{U}} \cdot \nabla \bar{\tau}+\nabla \cdot \mathbf{F}(\bar{\tau})=Q,
$$

where $\overline{\mathbf{U}}=(\overline{\mathbf{u}}, \bar{w})$ is the $3 \mathrm{D}$ mean velocity, $\mathbf{F}(\bar{\tau})=$ $\mathbf{F}_{H}(\bar{\tau})+\mathbf{e}_{z} F_{v}(\bar{\tau})$ is the 3D mesoscale-induced tracer flux, 
$\mathbf{e}_{z}=(0,0,1)$, and $Q$ represents external forcing. The where horizontal flux is given by

$$
\mathbf{F}_{H}(\bar{\tau})=-\kappa_{M} \cdot \nabla_{H} \bar{\tau}
$$

where $\kappa_{M}$ is the mesoscale diffusivity discussed below. The vertical tracer flux is given by

$$
\begin{aligned}
\mathbf{F}_{v}(\bar{\tau}) & =-\kappa_{M} \Phi \boldsymbol{\Omega}^{\|} \cdot\left(\nabla_{H} \bar{\tau}+\nabla_{\rho} \bar{\tau}\right)-\kappa_{M}(1-\Phi) \boldsymbol{\Omega} \cdot \nabla_{H} \bar{\tau}, \\
\mathbf{\Omega}^{\|} & =|\mathbf{s}|^{-2} \mathbf{s} \cdot \mathbf{\Omega} \mathbf{s},
\end{aligned}
$$

$$
\nabla_{\rho} \bar{\tau}=\nabla_{H} \bar{\tau}+\mathbf{s} \partial_{z}, \quad \Phi(z)=\frac{z^{2}}{h_{*}^{2}} \frac{N^{2}}{N_{*}^{2}}, \quad \Phi(0)=0,
$$

$\Phi\left(-h_{*}\right)=1$.

Here, $N$ is the Brunt-Väisälä frequency, $\mathbf{s}$ is the slope of the isopycnals, and $h_{*}$ denotes the depth of the D regime. The function $\Phi(z)$ allows one to match the flux at $h_{*}$ with that of the A regime. We have

Tracer: surface $F_{v}(\bar{\tau})=0 ;$ bottom D regime $F_{v}(\bar{\tau})=-\kappa_{M} \boldsymbol{\Omega}^{\|} \cdot\left(\nabla_{H} \bar{\tau}+\nabla_{\rho} \bar{\tau}\right)$

Buoyancy: surface $F_{v}(\bar{b})=0 ;$ bottom D regime $F_{v}(\bar{b})=-\kappa_{M} \boldsymbol{\Omega}^{\|} \cdot \nabla_{H} \bar{b}=\kappa_{M} N^{2} \boldsymbol{\Omega} \cdot \mathbf{s}$

$$
\begin{gathered}
\boldsymbol{\Omega}(z)=\left[\boldsymbol{\omega}(z) \times \mathbf{e}_{z}-\Phi(z) \boldsymbol{\omega}_{*} \times \mathbf{e}_{z}\right]+\Phi(z) \frac{N(z)^{2}}{N_{*}^{2}} \mathbf{s}(z) \\
f r_{d}^{2} \boldsymbol{\omega}(z) \equiv z \mathbf{u}_{D}-\int_{z}^{0} \mathbf{u}_{D}\left(z^{\prime}\right) d z^{\prime}, \quad \mathbf{u}_{D}=\overline{\mathbf{u}}-\mathbf{u}_{d},
\end{gathered}
$$

where and $r_{d}$ is the first Rossby deformation radius. In the case of buoyancy

$$
\text { Buoyancy: at } \quad z=-h_{*}, \quad \mathbf{\Omega}=\mathbf{s}, \quad F_{v}(\bar{b})=\kappa_{M} N^{2}\left|\mathbf{s}^{2}\right| .
$$

The last relation coincides with the form of the vertical buoyancy flux given by the GM model.

\section{2) A Regime}

The thickness-weighted (isopycnal) averages used to express the equations in this regime do not coincide with the Eulerian averages appropriate to the $\mathrm{D}$ regime. The different types of averages bring a new vector $\mathbf{E}$ in the mean tracer equation that now reads as follows:

$$
\begin{aligned}
\partial_{t} \bar{\tau}+\overline{\mathbf{U}} \cdot \nabla \bar{\tau}+\nabla \cdot \mathbf{F}(\bar{\tau}) & =Q, \\
\mathbf{F}(\bar{\tau}) & =\mathbf{F}_{\text {skew }}(\bar{\tau})+\mathbf{F}_{\text {redi }}(\bar{\tau})+\mathbf{E}(\bar{\tau}) .
\end{aligned}
$$

The skew flux is such that $\nabla \cdot \mathbf{F}_{\text {skew }}=\mathbf{U}^{+} \cdot \nabla \bar{\tau}$, where $\mathbf{U}^{+}=$ $\left(\mathbf{u}^{+}, w^{+}\right)$is the nondivergent, 3D eddy-induced velocity, and the Redi flux is $\mathbf{F}_{\text {redi }}=-\kappa_{M}\left(\nabla_{\rho} \bar{\tau}+\mathbf{s} \cdot \nabla_{\rho} \bar{\tau} \mathbf{e}_{z}\right)$. Last,

$$
\mathbf{E}=-\frac{r_{d}^{2}}{K} \frac{\partial K}{\partial z}\left(\mathbf{e}_{z} \times \mathbf{u}_{D} \cdot \nabla_{\rho} \bar{\tau}\right) \mathbf{e}_{z} \times \mathbf{s}
$$

where $K$ is the eddy kinetic energy. Because of the smallness of this term, it will be neglected hereinafter.

\section{3) EDDY-INDUCED VELOCITY}

The eddy-induced velocity is given by

$$
\mathbf{u}^{+}=\mathbf{u}_{\mathrm{GM}}^{+}-\frac{\kappa_{M}}{f r_{d}^{2}} \mathbf{e}_{z} \times \mathbf{c}_{R}+\frac{\kappa_{M}}{\sigma f r_{d}^{2}} \mathbf{e}_{z} \times\left(\overline{\mathbf{u}}-\mathbf{u}_{d}\right) .
$$

In compact form,

$$
\mathbf{u}^{+}=-\frac{\partial}{\partial z} \kappa_{M} \xi, \quad \kappa_{M}(z) \xi(z)=-\int_{-H_{b}}^{z} \mathbf{u}^{+}\left(z^{\prime}\right) d z^{\prime},
$$

where $\sigma \equiv \sigma_{t}\left(1+\sigma_{t}\right)^{-1}, \sigma_{t}$ is the turbulent Prandtl number of $O(1), \mathbf{c}_{R}=r_{d}^{2} \mathbf{e}_{z} \times \boldsymbol{\beta}, \boldsymbol{\beta}=\nabla f$ is the Rossby phase velocity, and $H_{b}$ is the ocean depth.

\section{4) DRIFT VELOCITY}

The drift velocity was derived to be

$$
\mathbf{u}_{d}(x, y)=\sigma \mathbf{c}_{R}+\langle\overline{\mathbf{u}}\rangle-\sigma f r_{d}^{2} \mathbf{e}_{z} \times\left(\left\langle\frac{\partial \mathbf{s}}{\partial z}\right\rangle-\frac{\mathbf{s}_{*}}{H_{*}}\right),
$$

where the average (angle brackets) is defined as follows:

$$
\langle\varphi\rangle=\frac{\int_{-H_{b}}^{-h_{*}} \varphi(z) \kappa_{M}(z) d z}{\int_{-H_{b}}^{-h_{*}} \kappa_{M}(z) d z}, \quad H_{*} \equiv \frac{\int_{-H_{b}}^{-h_{*}} \kappa_{M}(z) d z}{\kappa_{M}\left(h_{*}\right)}
$$

and in Eq. (A.12) $\mathbf{S}_{*}$ is the isopycnal slope at $h_{*}$. 


\section{b. Mesoscale diffusivity $\kappa_{M}$}

The mesoscale diffusivity is given by

$$
\kappa_{M}=\alpha r_{d} K^{1 / 2} \varpi\left(\mathbf{u}_{D}, K\right), \quad \varpi=\left(1+\frac{1}{K}\left|\mathbf{u}_{D}\right|^{2}\right)^{-1 / 2},
$$

where $\alpha \simeq 1 / 2$.

\section{c. Eddy kinetic energy}

The eddy kinetic energy is

$$
K(x, y, z)=\Gamma(x, y, z) K_{s}(x, y),
$$

where $\Gamma(x, y, z)$ is the vertical profile and $K_{s}(x, y)$ is the surface value. We have

$$
\Gamma(x, y, z)=\left|1+a_{0}\right|^{-2}\left|a_{0}+B_{1}(z)\right|^{2}, \quad a_{0}=\left|B_{1}\left(-H_{b}\right)\right|^{1 / 2} .
$$

Here, $B_{1}(z)$ is the first baroclinic mode solution of the eigenvalue problem

$$
\partial_{z z} \varphi+\left(N / f r_{d}\right)^{2} \varphi=0, \quad \varphi=N^{-2} \partial_{z} B_{1}
$$

with the boundary conditions $\partial_{z} B_{1}=0$ at $z=-H_{b}, 0$, and $B_{1}(0)=1$. The surface kinetic energy $K_{s}$ was derived to be

$$
\begin{aligned}
K_{s} & =\alpha_{K}^{-1}(1+\mathrm{BD})^{-1}\left(K_{A}+K_{D}\right), \\
\mathrm{BD} & \equiv\left(\int_{-H_{b}}^{0} \Gamma(z) d z\right)^{-1} \int_{-H_{b}}^{-h_{*}} \gamma(z) \Gamma(z) d z \\
\alpha_{K} & \equiv\left(C_{k} r_{d}\right)^{-1} \int_{-H_{b}}^{0} \Gamma^{3 / 2}(z) d z, \\
C_{K} & \equiv\left(\frac{3}{2} \mathrm{Ko}\right)^{3 / 2} \sigma_{t}^{1 / 2}, \quad 4 \leq \mathrm{Ko} \leq 8 \\
K_{A} & =\alpha r_{d} \int_{-H_{b}}^{-h_{*}} \varpi \Gamma^{1 / 2}(z) N^{2} \mathbf{s} \cdot \boldsymbol{\xi} d z, \\
K_{D} & =\alpha r_{d} \int_{-h_{*}}^{0} \varpi \Gamma^{1 / 2}(z) N^{2} \mathbf{s} \cdot \mathbf{\Omega} d z \\
\gamma(z) & =(2 / \pi)^{1 / 2}\left(H_{b} / \delta_{b}\right) \exp \left(-\zeta^{2} / 2\right), \\
\zeta & \equiv\left(z+H_{b}\right) \delta_{b}^{-1}, \quad \delta_{b}=40 \mathrm{~m},
\end{aligned}
$$

where Ko is the Kolmogorov constant.

\section{d. Depth of the D regime}

The depth of the $\mathrm{D}$ regime is given by

$$
h_{*}=0.5\left[\mathrm{HBL}+\max N^{2}(z)\right],
$$

where HBL, defined in Large et al. (1994, 371-372), is the depth at which the bulk Richardson number relative to the surface reaches values of $0.3-1$.

\section{e. Implementation in an OGCM}

The 3D mesoscale-induced tracer flux is written in the tensor form:

$$
\mathbf{F}(\bar{\tau})=-\kappa_{M} \mathbf{K} \cdot \nabla \bar{\tau},
$$

where

A regime: $\quad \mathbf{K}=\left(\begin{array}{ccc}1 & 0 & s_{x}-\xi_{x} \\ 0 & 1 & s_{y}-\xi_{y} \\ s_{x}+\xi_{x} & s_{y}+\xi_{y} & s^{2}\end{array}\right)$

D regime: $\mathbf{K}=\left(\begin{array}{ccc}1 & 0 & 0 \\ 0 & 1 & 0 \\ K_{31} & K_{32} & K_{33}\end{array}\right)$

$K_{31}=(1-\Phi) \Omega_{x}+2 \Phi \Omega_{x}^{\|}, \quad K_{32}=(1-\Phi) \Omega_{y}+2 \Phi \Omega_{y}^{\|}$,

$K_{33}=\Omega \cdot \mathbf{s}$.

\section{APPENDIX B}

\section{The OGCM}

We employed the 3D diffusivity tensor for an arbitrary tracer given in section 7 of $\mathrm{C} 18$, the mesoscale diffusivity Eq. (A.14), and the KPP vertical mixing scheme (Large et al. 1994) in the GISS ER-model which is the ocean component of the coupled NASA GISS model E (Russell et al. 1995, 2000; Liu et al. 2003). An early version of the revised E2-R code was run in a standalone mode (Danabasoglu et al. 2014). It employs a mass coordinate approximately proportional to pressure with 32 vertical layers with thickness from $\approx 12 \mathrm{~m}$ near the surface to $\approx 200 \mathrm{~m}$ at the bottom. The horizontal resolution is $1.25^{\circ}$ (longitude) by $1^{\circ}$ (latitude). It is a fully dynamic, non-Boussinesq, mass-conserving free-surface ocean model using a quadratic upstream scheme for the horizontal advection of tracers and a centered difference scheme in the vertical. An 1800-s time step is used for tracer evolution. Sea ice dynamics, thermodynamics, and ocean-sea ice coupling are represented as in the CMIP5 model-E configuration (Schmidt et al. 2014), save that here ice is on the ocean model grid. To force the model, we used the CORE-I Protocol (Griffies et al. 2009) with fluxes obtained from bulk formulae, the inputs to which are the ocean model surface state and atmospheric 
conditions derived from a synthesis of observations that repeat the seasonal cycle of a "normal year." The results we present correspond to the output of the final 20 winters (July-September) of a 500-yr run.

\section{REFERENCES}

Bates, M., R. Tulloch, J. Marshall, and R. Ferrari, 2014: Rationalizing the spatial distribution of mesoscale eddy diffusivity in terms of mixing length theory. J. Phys. Oceanogr., 44, 15231540, https://doi.org/10.1175/JPO-D-13-0130.1.

Buckingham, C. E., Z. Khaleel, A. Lazar, A. P. Martin, J. T. Allen, A. C. Naveira Garabato, A. F. Thompson, and C. Vic, 2017: Testing Munk's hypothesis for sub-mesoscale eddy generation using observations in the North Atlantic. J. Geophys. Res. Oceans, 122, 6725-6745, https://doi.org/10.1002/2017JC012910.

Canuto, V. M., and M. S. Dubovikov, 1996: A dynamical model for turbulence. General Formalism. Phys. Fluids, 8, 571-586, https://doi.org/10.1063/1.868842.

— 8, 1-30, https://doi.org/10.1016/j.ocemod.2003.11.003.

_- and —_, 2011: Comparison of four mixed layer mesoscale parameterizations and the equations for an arbitrary tracer. Ocean Modell., 39, 200-2007, https://doi.org/10.1016/ j.ocemod.2011.04.008

—-, Y. Cheng, M. S. Dubovikov, A. M. Howard, and A. Leboissetier, 2018a: Parameterization of mixed layer and deep-ocean mesoscales including nonlinearity. J. Phys. Oceanogr., 48, 555-572, https://doi.org/10.1175/JPO-D-160255.1.

,-- , and A. M. Howard, 2018b: Subduction by submesoscales. J. Geophys. Res. Oceans, 123, 8688-8700, https://doi.org/ 10.1029/2018JC014142.

,,--- , and M. S. Dubovikov, 2019: Three-dimensional, space-dependent mesoscale diffusivity: Derivation and implications. J. Phys. Oceanogr., 49, 1055-1074, https://doi.org/ 10.1175/JPO-D-18-0123.1.

Chelton, D. B., M. G. Schlax, and R. M. Samelson, 2011: Global observations of nonlinear mesoscale eddies. Prog. Oceanogr., 91, 167-216, https://doi.org/10.1016/J.POCEAN.2011.01.002.

Cushman-Roisin, B., 1987, Subduction. Dynamics of the Ocean Surface Mixed Layer: Proc. 'Aha Huliko'a Hawaiian Winter Workshop, Honolulu, HI, University of Hawai'i at Mānoa, 181-196, http://www.soest.hawaii.edu/PubServices/1987pdfs/ Cushman_Roisin.pdf.

Danabasoglu, G., and Coauthors, 2014: North Atlantic simulations in Coordinated Ocean-Reference Experiment phase II (CORE-II). Part I: Mean states. Ocean Modell., 73, 76-107, https://doi.org/10.1016/j.ocemod.2013.10.005.

Ferrari, R., and K. L. Polzin, 2005, Finescale structure of the T-S relation in the eastern North Atlantic. J. Phys. Oceanogr., 35, 1437-1454, https://doi.org/10.1175/JPO2763.1.

Gent, P. R., 2016: Effects of Southern Hemisphere wind changes on the meridional overturning circulation. Annu. Rev. Mar. Sci., 8, 79-94, https://doi.org/10.1146/annurev-marine122414-033929.

_ , and J. C. McWilliams, 1990: Isopycnal mixing in ocean circulation models. J. Phys. Oceanogr., 20, 150-155, https://doi.org/ 10.1175/1520-0485(1990)020<0150:IMIOCM > 2.0.CO;2.

Gregory, J. M., 2000: Vertical heat transports in the ocean and their effect on time dependent climate change. Climate Dyn., 16, 501-515, https://doi.org/10.1007/s003820000059.
Griffies, S. M., and Coauthors, 2009: Coordinated Ocean-ice Reference Experiments (COREs). Ocean Modell., 26, 1-46, https://doi.org/10.1016/j.ocemod.2008.08.007.

Gnanadesikan, A., S. M. Griffies, and B. L. Samuels, 2007: Effects in a climate model of slope tapering in neutral physics schemes. Ocean Modell., 16, 1-16, https://doi.org/10.1016/ j.ocemod.2006.06.004.

Hiraike, Y., Y. Tanaka, and H. Hasumi, 2016: Subduction of Pacific Antarctic Intermediate Water in an eddy-resolving model. J. Geophys. Res. Oceans, 121, 133-147, https://doi.org/10.1002/ 2015JC010802.

Klocker, A., and D. Marshall, 2014: Advection of baroclinic eddies by depth mean flow. Geophys. Res. Lett., 41, 3517-3521, https://doi.org/10.1002/2014GL060001.

Large, W. G., J. C. McWilliams, and S. C. Doney, 1994: Oceanic vertical mixing: A review and a model with a nonlocal boundary layer parameterization. Rev. Geophys., 32, 363-403, https://doi.org/10.1029/94RG01872.

Liu, J., G. A. Schmidt, D. G. Martinson, D. Rind, G. L. Russell and X. Yuan 2003, Sensitivity of sea ice to physical parameterizations in the GISS global climate model. J. Geophys. Res., 108, 3053, https://doi.org/10.1029/2001JC001167.

Luneva, M. V., C. A. Clayson, and M. S. Dubovikov, 2015: Effects of mesoscale eddies in an active mixed layer: Test of the parameterization in eddy resolving simulations. Geophys. Astrophys. Fluid Dyn., https://doi.org/10.1080/ 03091929.2015.1041023.

Marshall, D., 1997: Subduction of water masses in an eddying ocean. J. Mar. Res., 55, 201-222, https://doi.org/10.1357/ 0022240973224373.

Mazloff, M. R., P. Heimbach, and C. Wunsch, 2010: An eddypermitting Southern Ocean state estimate (SOSE). J. Phys. Oceanogr., 40, 880-889, https://doi.org/10.1175/2009JPO4236.1.

Mensa, J. A., Z. Garraffo, A. Griffa, T. M. Ozgokmen, A. Haza, and M. Veneziani, 2013: Seasonality of submesoscale dynamics in the Gulf Stream region. Ocean Dyn., 63, 923-941, https://doi.org/10.1007/s10236-013-0633-1.

Ramachandran, S., A. Tandon, and A. Mahadevan, 2014: Enhancement in vertical fluxes at a front by mesoscale-submesoscale coupling. J. Geophys. Res. Oceans, 119, 8495-8511, https:// doi.org/10.1002/2014JC010211.

Russell, G. L., J. R. Miller, and D. H. Rind, 1995: A coupled atmosphereocean model for transient climate change. Atmos.-Ocean, 33, 683-730, https://doi.org/10.1080/07055900.1995.9649550.

R. A. Ruedy, G. A. Schmidt, and S. Sheth, 2000: Comparison of model and observed regional temperature changes during the past $40+$ years. J. Geophys. Res., 105 , 14 891-14 898, https://doi.org/10.1029/2000JD900156.

Sallée, J. B., and S. R. Rintoul, 2011, Parameterization of eddy-induced subduction in the Southern Ocean surface layer. Ocean Modell., 39, 146-153, https://doi.org/10.1016/J.OCEMOD.2011.04.001.

- K. Speer, R. Morrow, and R. Lumpkin, 2008: An estimate of Lagrangian eddy statistics and diffusion in the mixed layer of the Southern Ocean. J. Mar. Res., 66, 441-463, https://doi.org/ 10.1357/002224008787157458.

,-- S. Rintoul, and S. Wijffels, 2010: Southern Ocean thermocline ventilation. J. Phys. Oceanogr., 40, 509-529, https:// doi.org/10.1175/2009JPO4291.1.

Scharffenberg, M. G., and D. Stammer, 2010: Seasonal variations of the geostrophic flow field and of eddy kinetic energy inferred from TOPEX/Poseidon and Jason-1 Tandem Mission Data. J. Geophys. Res., 115, C02008, https://doi.org/10.1029/ 2008JC005242. 
Schmidt, G. A., and Coauthors, 2014: Configuration and assessment of the GISS ModelE2 contributions to the CMIP5 archive. J. Adv. Model. Earth Syst., 6, 141-184, https://doi.org/ 10.1002/2013MS000265.

Sloyan, B., and S. Rintoul, 2001: Circulation, renewal and modification of Antarctic Mode Water and Intermediate Water. J. Phys. Oceanogr., 31, 1005-1030, https://doi.org/10.1175/ 1520-0485(2001)031<1005:CRAMOA > 2.0.CO;2.

Veneziani, M., A. Griffa, Z. Garrafo, and J. A. Mensa, 2014: Barrier layers in the tropical South Atlantic: Mean dynamics and sub-mesoscale effects. J. Phys. Oceanogr., 44, 265-288, https://doi.org/10.1175/JPO-D-13-064.1.
Visbeck, M., J. Marshall, T. Haine, and M. Spall, 1997: Specification of eddy transfer coefficients in coarse resolution ocean circulation models. J. Phys. Oceanogr., 27, 381-402, https://doi.org/10.1175/1520-0485(1997)027<0381:SOETCI> 2.0.CO;2.

WOCE, 2002: WOCE Global Data, version 3.0. WOCE Rep. 180/02, WOCE International Project Office, https://www.nodc.noaa.gov/ woce/woce_v3/wocedata_1/diu/welcome.htm.

Wunsch, C., 1997: The vertical partition of oceanic horizontal kinetic energy. J. Phys.Oceanogr., 27, 1770-1794, https://doi.org/10.1175/1520-0485(1997)027<1770:TVPOOH $>$ 2.0.CO;2. 